Acta Cryst. (2002). A58 (Supplement), C102

THE X-RAY CRYSTAL STRUCTURE OF FORMALDEHYDE DISMUTASE AT 2.3Å RESOLUTION

T. Hasegawa $^{1}$ A. Yamano ${ }^{1}$ K. Miura ${ }^{2}$ Y. Katsube ${ }^{2}$ H. Yanase ${ }^{3}$ N. Kato ${ }^{4}$ ${ }^{1}$ Rigaku Corporation X-Ray Research Laboratory 3-9-12 Matsubara-Cho, Akishima-Shi TOKYO 196-8666 JAPAN ${ }^{2}$ Japan Synchrotron Radiation Research Institute (JASRI), 1-1-1 Kouto, Mikazuki-cho, Sayo-gun, Hyogo, 679-5198, Japan ${ }^{3}$ Department of Biotechnology, Faculty of Engineering, Tottori University, 4-101 Koyama-Minami, Tottori, 680-8550, Japan ${ }^{4}$ Division of Applied Life Sciences, Graduate School of Agriculture, Kyoto University, Kitashirakawa Oiwake-Cho, Sakyo-Ku, Kyoto, 606-8502, Japan

Formaldehyde dismutase (FDM) exhibits the catalysis of the dismutation of aldehydes and alcohol: aldehyde oxdoreduction in the absence of an exogenous electron acceptor. The crystal structure of FDM was determined at $2.3 \AA$ resolution by multi-wavelength anomalous dispersion method. FDM is composed of four identical subunits with a molecular weight of 44,000. Each subunit contains 2 zinc atoms. Preliminary intensity data were collected using $\mathrm{CuK} \alpha$ radiation from a rotating-anode $\mathrm{X}$-ray generator (Rigaku ultraX $18 \mathrm{HF}$ ) operated at $40 \mathrm{kV} 100 \mathrm{~mA}$. Crystals of FDM belong to the space group I41 with unit-cell dimensions of $\mathrm{a}=\mathrm{b}=89.74 \AA \mathrm{c}=226.77 \AA$ and there are two molecules per asymetric unit. Intensity data of native crystal and anomalous dispersion data were collected using Synchrotron Radiation at SPring-8 BL38B1. Experimental phases were obtained with MAD method using 3 wavelengths.

Keywords: CRYSTAL STRUCTURE FORMALDEHYDE DISMUTASE OXIDOREDUCTASE
Acta Cryst. (2002). A58 (Supplement), C102

\section{STRUCTURES OF THE TWO POLYMORPHIC FORMS OF HUMAN HISTAMINE METHYLTRANSFERASE}

J.R. Horton K. Kawada X. Cheng

Emory University School of Medicine Biochemistry Dept. Rollins Research Building, G239 1510 Clifton Road ATLANTA GA 30322 USA

Histamine is an important messenger in cell-to-cell communication: it mediates allergy and inflammation, regulates gastric acid secretion, and is a neurotransmitter that affects several (patho)physiological processes. Histamine methyltransferase (HNMT) inactivates histamine by transferring a methyl group from S-adenosyl-L-methionine (AdoMet) to the ring nitrogen atom $\mathrm{Ne}_{2}$ of its substrate's imidazole yielding methylhistamine and S-adenosyl-Lhomocysteine. The less frequent polymorphic form (allozyme) of human HNMT (Ile105) has a lower activity (84\%) when compared to the Thr105 variant. The $10 \%$ of the population with the Ile105 allozyme have a greater risk for asthma.

The structure of HNMT has two domains: the larger is a consensus AdoMetbinding domain found in many other AdoMet-dependent methyltransferases, and a smaller domain reminiscent of the $\beta$-subdomain found in the bacterial CheR protein MTase involved in protein-protein interactions. The position of the allozyme variation is not near the active site but at the carboxyl end of a helix whose amino end is involved with binding AdoMet. HNMT has a deep catalytic cavity located at the domain interface. This cavity allows HNMT to be very selective for histamine while permitting it to accommodate inhibitors diverse in chemical structure as well as clinical pharmacology. Besides several antimalarial drugs such as amodiaquine and quinacrine, the anticholinesterase tacrine and the oncolytic agent metoprine are very potent inhibitors. Other inhibitors include the compound SKF-91488 and first generation antihistamines such as diphenhydramine. Structures of complexes of both human allozymes of HNMT, with many of these inhibitors, have been determined. These structures show several modes of binding to and inhibition of HNMT, and have implications for design of drugs for histamine-related diseases.

\section{Keywords: METHYLTRANSFERASES, DRUG INTERACTION, SINGLE NUCEOTIDE POLYMORPHISMS}

\section{Acta Cryst. (2002). A58 (Supplement), C102}

CRYSTAL STRUCTURE OF A BACTERIAL TRNA-PRECURSOR PROCESSING ENZYME: RNASE PH FROM BACILLUS SUBTILIS A. Kadziola ${ }^{1}$ L. S. Harlow ${ }^{2}$ K.-F. Jensen ${ }^{2}$ S. Larsen

${ }^{1}$ Centre For Crystallographic Studies Department of Chemistry, University of Copenhagen Universitetsoarken 5 COPENHAGEN DK-2100 DENMARK ${ }^{2}$ Department of Biological Chemistry, University of Copenhagen, Denmark

In Escherichia coli, the maturation of precursor tRNA molecules is carried out by multiple ribonucleases showing overlapping activities [1]. The exoribonuclease RNase $\mathrm{PH}$ is found to be involved in processing at the 3 ' end, cleaving the +2 nucleotide following the well-characterized tRNA-CCA sequence. A homologous protein has been detected in Bacillus subtilis, which has also been assigned RNase PH. In vitro, RNase PH catalyses the phosphorolysis of polyadenylate and the polymerization of nucleotide diphosphates into a tRNA chain. The phosphorolysis reaction has a requirement of inorganic phosphate and $\mathrm{Mg}^{2+}$, whereas the synthetic reaction only requires $\mathrm{Mg}^{2+}$. RNase $\mathrm{PH}$ from Bacillus subtilis has been crystallized with ammonium sulphate at $\mathrm{pH} 8.5$ in the presence of $\mathrm{Cd}^{2+}$. It crystallizes in space group $P 2_{1} 2_{1} 2_{1}$ with cell dimensions: $\mathrm{a}=100, \mathrm{~b}=146, \mathrm{c}=152 \AA$. The structure has been solved by isomorphous replacement using 5 derivatives containing from 2 to $6 \mathrm{Hg}$-sites. The crystal contains a 32 hexamer in the asymmetric unit. 242 residues of 245 in the monomer have been modeled. The $\mathrm{C}$-terminal Glu243-Lys245 is disordered. Residual electron density showed the presence of two sulphate ions per monomer. The hexamer of RNase $\mathrm{PH}$ is to be considered as a trimer of dimers. The dimer is structural homologous to the monomer of polynucleotide phosphorylase[2].

References

[1] Li \& Deutsher (1996) Cell 86, 503-512.

[2] Symmons, Jones \& Luisi (2000) Structure 8, 1215-1226. 